\title{
Fast immunosensing technique to detect Legionella pneumophila in different natural and anthropogenic environments: comparative and collaborative trials
}

\author{
Begoña Bedrina', Sonia Macián ${ }^{1}$, Inmaculada Solís², Roberto Fernández-Lafuente ${ }^{3}$, Eva Baldrich ${ }^{4}$ \\ and Guillermo Rodríguez ${ }^{* *}$
}

\begin{abstract}
Background: Legionellosis is an uncommon form of pneumonia. After a clinical encounter, the necessary antibiotic treatment is available if the diagnosis is made early in the illness. Before the clinical encounter, early detection of the main pathogen involved, Legionella pneumophila, in hazardous environments is important in preventing infectious levels of this bacterium. In this study a qualitative test based on combined magnetic immunocapture and enzyme-immunoassay for the fast detection of Legionella pneumophila in water samples was compared with the standard method, in both comparative and collaborative trials. The test was based on the use of anti-Legionella pneumophila antibodies immobilized on magnetic microspheres. The final protocol included concentration by filtration, resuspension and immunomagnetic capture. The whole assay took less than 1 hour to complete.

Results: A comparative trial was performed against the standard culture method (ISO 11731) on both artificially and naturally contaminated water samples, for two matrices: chlorinated tap water and cooling tower water.

Performance characteristics of the test used as screening with culture confirmation resulted in sensitivity, specificity, false positive, false negative, and efficiency of $96.6 \%, 100 \%, 0 \%, 3.4 \%$, and $97.8 \%$, respectively. The detection limit at the level under which the false negative rate increases to 50\% (LOD50) was 93 colony forming units (CFU) in the volume examined for both tested matrices. The collaborative trial included twelve laboratories. Water samples spiked with certified reference materials were tested. In this study the coincidence level between the two methods was $95.8 \%$.

Conclusion: Results demonstrate the applicability of this immunosensing technique to the rapid, simple, and efficient detection of Legionella pneumophila in water samples. This test is not based on microbial growth, so it could be used as a rapid screening technique for the detection of L. pneumophila in waters, maintaining the performance of conventional culture for isolation of the pathogen and related studies.
\end{abstract}

Keywords: Legionella, Detection, Immunosensing, Magnetic particles

\footnotetext{
* Correspondence: guiller@biotica.es

'Biótica, Bioquímica Analítica, S.L, Science and Technology Park of Jaume I University, Campus Riu Sec - Espaitec 2, planta baja, E12071, Castellón de la Plana, Spain

Full list of author information is available at the end of the article
} 


\section{Background}

Legionella pneumophila is the major cause of sporadic cases and outbreaks of legionellosis (91.5\%), with serogroup 1 being the predominant serotype (84.2\%) [1,2], among the 52 species and 70 sero-groups included in the genus Legionella [3-5]. Outbreaks of L. pneumophila occur throughout the world impacting public health as well as various industrial, tourist, and social activities [6]. Patients with immuno-compromised status are particularly susceptible to this atypical pneumonia [7]. This pathogen is present in both natural [6] and man-made [7] water environments like cooling towers, evaporative condensers, humidifiers, potable water systems, decorative fountains and wastewater systems (risk facilities). Human infection can occur by inhalation of contaminated aerosols [8]. Colonization at human-made water systems has been associated with biofilms yielding only some free bacterial cells $[1,9,10]$. Moreover, rapid fluctuations of the concentration of L. pneumophila at risk facilities have been reported [11], as well as persistence of L. pneumophila in drinking water biofilms mostly in a viable but non-culturable state (VBNC) [12], which has also been confirmed even after treatments with chlorine used to disinfect cooling towers [13,14]. In fact, $L$. pneumophila becomes non-culturable in biofilms in doses of $1 \mathrm{mg} / \mathrm{L}$ of monochloramine, making culture detection of this pathogen ineffective [15]. The effectiveness of treatments on Legionella pneumophila (chlorine, heat, ozone, UV, monochloramine) has been mainly evaluated based simply on cultivability and that could not be a real indicative of the absence of intact viable cells [16-18].

Official methods for Legionella detection are based on the growth of the microorganism in selective media $[19,20]$. At least 7 to 15 days are required for obtaining results due to the slow growth rate of the bacterium. Culture detection also shows low sensitivity, loss of viability of bacteria after collection, difficulty in isolating Legionella in samples contaminated with other microbial and the inability to detect VBNC bacteria [21]. Therefore, the development of a rapid and specific detection method for L. pneumophila monitoring and in real time would be crucial for the efficient prevention of legionellosis. Polymerase chain reaction (PCR) methods have been described as useful tools for L. pneumophila detection [22,23]. PCR reportedly provides high specificity, sensitivity, and speed, low detection limits and the possibility to quantify the concentration of the microorganisms in the samples using real-time PCR. However, it requires sophisticated and expensive equipment, appropriate installations and trained personnel [24]. PCR inhibiting compounds present in environmental samples may cause false negatives. Inhibition control is strongly recommended in those cases. Samples having inhibition must be diluted and retested. False positives can be caused by the inability of PCR to differentiate between cells and free DNA [25]. Finally, the cell number assigned to a certain amount of target genes varies by one order of magnitude depending on the growth phase and bacteria species, limiting the capability of PCR test for accurate bacterial quantification [26]. Immunodetection has provided the basis for the development of powerful analytical tools for a wide range of targets. During the last years, the number of publications in this field has increased significantly [27]. Traditionally, the most common method applied to microorganism detection has been the enzyme-linked immunosorbent assay (ELISA). The main drawback of ELISA is the high detection limit generated; which is often between $10^{5}$ and $10^{6} \mathrm{CFU} / \mathrm{mL}$ [28]. This limit may be improved to $10^{3}$ and $10^{4}$ cells $/ \mathrm{mL}$ using more sensitive detection methods [29,30]. The immobilization of antibodies onto the surface of magnetic beads to obtain immunomagnetic beads (IMB) has promoted the development of immunomagnetic separation (IMS). Thereby, IMS provides a simple but powerful method for specific capture, recovery and concentration of the desired microorganism from heterogeneous bacterial suspension [23,31-34].

A test based on IMS by anti-L. pneumophila immunomodified magnetic beads (LPMB), coupled to enzymelinked colorimetric detection has been proposed for the rapid detection of $L$. pneumophila cells in water samples [35]. In this study, intensive comparison of this immunomagnetic method (IMM) with the culture method is presented.

\section{Results}

\section{Comparative trial with natural samples}

The IMM test was applicable to detection of $L$. pneumophila in water samples. A total of 459 water samples, comprising both naturally contaminated and artificially contaminated samples were examined for the presence of $L$. pneumophila using the reference culture method (ISO 11731-Part 1) and the IMM test in parallel. The parameters for this comparison study were calculated from the results summarized in Table 1 as it is described in the Methods section. Sensitivity and specificity were estimated as $96.6 \%(284 / 294)$ and $88 \%$ (145/ 165), respectively for the IMM. This means that a proportion of actual positives and negatives are correctly assigned by the IMM test. False positives and false negatives were estimated as, respectively, $12.0 \%(20 / 304)$ and $3.4 \%(10 / 294)$. Some "false" positives could be related to problems in the culture method, as stated in the background that presents some limitations under different circumstances [12,15,21]. In fact, the PCR analysis of some of the samples initially considered false positives confirmed later the existence of DNA from L. pneumophila 
Table 1 Comparison of the immunomagnetic method with the standard culture method

\begin{tabular}{ccccc}
\hline & \multicolumn{4}{c}{$\begin{array}{c}\text { Immunomagnetic method (IMM) as } \\
\text { screening assay }\end{array}$} \\
\hline & $\begin{array}{c}\text { Without } \\
\text { confirmation }\end{array}$ & With confirmation \\
bulture method (ISO 11731) & + & - & + & - \\
+ & 284 & 10 & 284 & 10 \\
- & 20 & 145 & 0 & 165 \\
\hline
\end{tabular}

a Presumptive IMM results.

${ }^{\mathrm{b}}$ Confirmed IMM results.

in those samples (results not shown), suggesting a failure of the culture method. From the point of view of the IMM as a screening test with culture confirmation, presumptive test negative results can be added to the true negatives. In this case sensitivity and specificity were estimated as, respectively, 96.6\% (284/294) and 100\% (0/165) for the IMM. False positives and false negatives were estimated as, respectively, $0 \%(0 / 324)$ and $3.4 \%(10 / 294)$. The low false negative ratio suggests that the IMM is very reliable.

Efficiency of the IMM as screening assay without confirmation was estimated as $93.5 \%$ (429/459). The IMM with confirming culture method had an efficiency of $97.8 \%$. This means that results obtained with the IMM test exhibited a high agreement with the reference culture method.

\section{Detection limit}

The detection limit of the IMM test was determined by testing water samples spiked with different $L$. pneumophila (ATCC 33152) concentrations at 5 different levels (Table 2). The detection limit was defined as the lowest number of cultivable $L$. pneumophila organisms (confirmed by culture) that can be detected with a probability of $50 \%$. On the basis of this criterion, the detection limit of IMM for L. pneumophila was determined as $93 \mathrm{CFU}$ per volume examined for the studied matrices. Here the volume examined is the filtered volume of the original water sample.

Table 2 Summary of immunomagnetic test and ISO reference method results for the estimation of $L \mathrm{D}_{50}$

\begin{tabular}{ccc}
\hline $\begin{array}{c}\text { Level } \\
\text { no. }\end{array}$ & $\begin{array}{c}\text { Culture count, } \\
\text { CFU/mL }\end{array}$ & $\begin{array}{c}\text { IMM presumptive positive/total } \\
\text { portions tested }\end{array}$ \\
\hline 1 & 0 & $0 / 6$ \\
2 & 3.4 & $0 / 10$ \\
3 & 15.1 & $14 / 30$ \\
4 & 20.4 & $7 / 10$ \\
5 & 68.3 & $10 / 10$ \\
\hline
\end{tabular}

\section{Collaborative trial}

Table 3 shows the results of the eleven accepted laboratories that have evaluated the IMM test. The concentrations estimated by the color chart of the IMM test were highly coincident with the reported culture results for each one of the three groups of samples prepared with certified reference material (pills) containing $L$. pneumophila. For the two pills used as negative control, not having $L$. pneumophila, this bacterium was not detected by any of the two methods (culture isolation and IMM test) in any of the participating laboratories. Coincidence between both methods was of $95.8 \%$. Comparison gave good results, with clear coincidence with the standard culture method but a higher rate of analysis.

\section{Discussion}

This study confirms the suitability of the IMM test for the detection of $L$. pneumophila in water samples. The final protocol comprised sample pre-concentration by filtration and resuspension, magnetic capture using immunoactivated beads, and colorimetric enzyme-linked immunodetection in just $1 \mathrm{~h}$ of analysis, while the standard protocol requires 7-14 days. Sensitivity (96.6\%), specificity (100\%), false positives (0\%), false negatives (3.4\%), and efficiency (97.8\%) were determined. The LOD50 was only $93 \mathrm{CFU}$ of $L$. pneumophila in the volume examined for the selected matrices, which is significantly below the values reported for other conventional methods such as ELISA. This occurs even though some of the samples (mainly from cooling towers) presented viscosity and dirtiness that made handling difficult.

\section{Conclusions}

In view of these results, the IMM test could be a valuable tool for the rapid, simple and robust detection of free L. pneumophila at risk installations, in a weekly and even daily basis, contributing to minimize the risk of outbreaks by this pathogen. At theses environments, presence of $L$. pneumophila or a high percentage of positive points, have been identified as factors contributing to explain case onset [36]. The reported combination of magnetic capture and enzyme-immunoassay provides a user-friendly and extremely easy to use assay format, which is a valuable low-cost tool for the implementation of in situ surveillance, development of Water Safety Plans, or fast screening of water samples. In combination with other established techniques, such culture and PCR, addressed to isolation and identification of $L$. pneumophila, IMM could be useful for an integral surveillance. From the results presented in this study, Legipid IMM test is a very promising tool to fight against legionellosis and similar configurations could be used to detect other dangerous pathogens. 
Table 3 Legionella pneumophila determination in collaborative trial, Log (CFU/9 mL) (by participant no.) ${ }^{a}$

\begin{tabular}{|c|c|c|c|c|c|c|c|c|c|c|c|c|c|c|c|c|c|c|c|c|c|c|c|c|c|c|c|c|c|c|c|c|c|c|c|c|}
\hline \multirow{3}{*}{$\begin{array}{l}\text { Level of spiking } \\
\log _{10} \text { CFU/9 mL }\end{array}$} & \multirow{3}{*}{ Pill } & \multicolumn{11}{|c|}{ Culture results } & \multicolumn{24}{|c|}{ Immunomagnetic results } \\
\hline & & \multicolumn{11}{|c|}{ Culture count $\log _{10} \mathrm{CFU} / 9 \mathrm{~mL}^{\mathrm{c}}$} & \multicolumn{12}{|c|}{ Estimated magnitude order $\log _{10} \mathrm{CFU} / 9 \mathrm{~mL}$} & \multicolumn{12}{|c|}{ Qualitative results $^{d}$} \\
\hline & & 1 & 2 & 3 & 4 & 5 & 6 & 7 & 8 & 9 & 10 & 11 & 1 & 2 & & 3 & 4 & 5 & 6 & 7 & 8 & 9 & 10 & 11 & 1 & 2 & 3 & 4 & 5 & 6 & 7 & 8 & 89 & & 101 & 11 \\
\hline \multirow[t]{2}{*}{0} & $P_{6}$ & ND & ND & ND & ND & ND & ND & ND & ND & ND & ND & ND & ND & ND & & ID & ND & ND & ND & ND & ND & ND & ND & ND & A & A & A & A & - A & A & - A & A & $A \quad A$ & & A & A \\
\hline & $P_{8}$ & ND & ND & ND & ND & ND & ND & ND & ND & ND & ND & ND & ND & ND & & ID & ND & ND & ND & ND & ND & ND & ND & ND & A & A & A & A & A & A & - A & & $A \quad A$ & & A & A \\
\hline \multirow[t]{2}{*}{2.23} & $\mathrm{P}_{4}$ & 2.83 & 2.22 & 2.21 & 2.47 & 2.57 & 2.11 & 2.38 & 2.23 & 2.73 & 1.98 & 2.32 & 3.0 & $<3$. & & 0 & $<3.0$ & $<3.0$ & $<3.0$ & 2.0 & 2.0 & 3.0 & 2.0 & 3.0 & P & $P$ & $P$ & P & $P$ & P & P & & $P \quad P$ & & $P$ & $P$ \\
\hline & $P_{7}$ & 2.11 & 2.16 & 2.36 & 2.25 & 2.13 & 2.11 & 2.10 & 2.01 & 2.17 & 1.90 & 2.32 & $<4.0$ & $<3$. & & 4.0 & $<4.0$ & $<3.0$ & 3.0 & 3.0 & 2.0 & $<4.0$ & 2.0 & 3.0 & P & $P$ & $P$ & $\mathrm{P}$ & $P$ & P & P & & $P \quad P$ & & $P$ & $P$ \\
\hline \multirow[t]{2}{*}{2.88} & $P_{1}$ & 3.07 & 2.86 & 3.12 & 3.19 & 3.04 & 1.99 & 2.99 & 2.96 & 2.69 & 2.78 & 2.85 & 4.0 & 3.0 & & 0 & $<4.0$ & 3.0 & 3.0 & 3.0 & 3.0 & 3.0 & 3.0 & 3.0 & P & $P$ & $P$ & $P$ & $P$ & P & P & & $P \quad P$ & & $P$ & $P$ \\
\hline & $P_{3}$ & 3.13 & 2.90 & 3.11 & 3.13 & 3.07 & 2.29 & 2.61 & 2.51 & 2.77 & 2.57 & 2.77 & 3.0 & 3.0 & & 0 & $<4.0$ & 3.0 & 2.0 & 3.0 & 2.0 & $<4.0$ & 3.0 & 3.0 & P & $P$ & $P$ & $P$ & $P$ & P & P & P & $P \quad P$ & & $P$ & P \\
\hline \multirow[t]{3}{*}{3.82} & $P_{2}$ & 4.01 & 4.23 & 4.03 & 3.93 & 3.76 & 3.59 & 3.49 & 3.56 & 3.21 & 3.59 & 4.22 & 4.0 & $>4$. & & 4.0 & $<4.0$ & 4.0 & $>4.0$ & 4.0 & 3.0 & 4.0 & $>4.0$ & 4.0 & P & $P$ & $P$ & $\mathrm{P}$ & $P$ & P & P & P & $P \quad P$ & & $P$ & $P$ \\
\hline & $P_{5}$ & 3.92 & 4.20 & 4.30 & 3.64 & 3.63 & 3.94 & 3.77 & 3.66 & 3.97 & 3.61 & 3.95 & 4.0 & 4.0 & & 1.0 & 4.0 & 4.0 & $>4.0$ & 4.0 & 3.0 & 4.0 & $>4.0$ & $>4.0$ & $P$ & $P$ & $P$ & P & $P$ & P & P & P & $P \quad P$ & & P & $P$ \\
\hline & $P_{9}$ & 3.33 & 4.20 & 3.91 & 3.89 & 3.92 & 3.71 & 3.48 & 3.63 & 3.97 & 2.91 & 3.99 & 4.0 & 4.0 & & 1.0 & 4.0 & 4.0 & $>4.0$ & $>4.0$ & $>4.0$ & 3.0 & 4.0 & 4.0 & P & $P$ & $P$ & $P$ & $P$ & P & P & F & $P \quad P$ & & P & P \\
\hline
\end{tabular}

${ }^{a}$ This table includes only results from participating laboratories that were not excluded due to obvious deviation from the trial protocol.

${ }^{\mathrm{b}}$ Concentrations calculated from the results provided by the 11 participating laboratories, assigned to the used reference materials (pills).

${ }^{\mathrm{d}} \mathrm{A}$, absence; $\mathrm{P}$, presence. 


\section{Methods}

\section{Comparative trial}

Intensively water testing was made to compare the IMM to the ISO 11731 reference culture method. Tap, cooling tower and natural water samples were collected from different locations and different seasons during three years in the period 2008-2011. A total number of 459 water samples were tested. From these samples, 189 were naturally contaminated samples and 270 were artificially contaminated samples. Distribution of naturally contaminated samples was the following: 84 samples from cooling towers, 94 samples from tap water, 8 samples from water wells and 3 waste water samples. Distribution of artificially contaminated samples was the following: 104 samples from cooling towers, 166 samples from tap water. Both the collection L. pneumophila strain (ATCC 33152) and an environmental isolate of $L$. pneumophila sg 1 were used as inoculums to prepare artificially contaminated samples. Legionella pneumophila was grown for 3 days on BCYE agar (Buffered Charcoal Yeast Extract) supplemented with glycine, vancomycin, polymixine and cycloheximide (GVPC medium) to obtain exponential-phase cultures. These cultures were used to inoculate water samples. Each sample was tested for the level of background flora by standard plate count of dilutions series of each type of sample. The concentration of Legionella pneumophila ranged from $10^{2} \mathrm{CFU}$ to $10^{7} \mathrm{CFU}$ in the volume examined, between $0.1 \mathrm{~L}$ to $1.0 \mathrm{~L}$ (usually $1.0 \mathrm{~L}$ ). Generally, the level of total bacterial counting was below $50 \mathrm{CFU} / \mathrm{mL}$ for the tap water samples, and this level was ranging from $10^{2}$ to $10^{5}$ $\mathrm{CFU} / \mathrm{mL}$ for cooling tower water samples, most of them between $10^{3}$ and $10^{4} \mathrm{CFU} / \mathrm{mL}$. Each of these examined volumes were concentrated by filtration through 0.4$\mu \mathrm{m}$-pore-size, 47-mm-diameter polycarbonate sterile membranes (Sartorius, Germany), following the instructions of the International Standard method ISO11731Part 1. After filtration, each membrane was directly placed in a screw cap sterile container containing $10 \mathrm{~mL}$ of the reagent L0 (Biótica, Spain). Then L. pneumophila was eluted by vortex mixing for $2 \mathrm{~min}$. An average of $47 \%$ of the seeded L. pneumophila organisms were recovered by filtration. This concentrate represented the prepared sample. The volume of this sample was divided into two portions: $9 \mathrm{~mL}$ for IMM test and $1 \mathrm{~mL}$ for the culture test. The positivity or negativity of the water samples by the IMM was visually recorded by the colorimetric end-point reaction.

\section{Detection limit}

The detection limit was determined considering validation protocols of international certification bodies $[37,38]$. Both tap and cooling tower waters were collected and tested negative for the L. pneumophila before its use as matrices. Legionella pneumophila sg 1 (ATCC 33152, Laboratoire BioRéférence, ipl-Groupe, France) was resuspended into $20 \mathrm{~mL}$ of a sterile saline solution at room temperature under gently agitation. These 20 mL-suspensions were used to inoculate one liter of selected matrices. Five levels of target contamination were prepared to obtain fractional positive results by the IMM method. Level one corresponded to absence of the target organism, level two corresponded to a proportion of IMM positive results minor than 50\%, level three corresponded to a proportion of IMM positive results around the $50 \%$, level four corresponded to a proportion of IMM positive results higher than 50\%, and finally level five corresponded to a proportion of IMM positive results of $100 \%$.

These artificially contaminated $1.0 \mathrm{~L}$-samples left to equilibrate for $15-16$ hours at $4^{\circ} \mathrm{C}$ prior starting analysis, to stabilize the inoculated target organism. Each $1.0 \mathrm{~L}-$ sample was then divided into ten $100 \mathrm{~mL}$-aliquots as replicates. A total of $66100 \mathrm{~mL}$-aliquots were examined. Each of these $100 \mathrm{~mL}$-aliquots was concentrated by filtration following the instructions of the International Standard Method ISO11731-Part 1. The volume of each $10 \mathrm{~mL}$-concentrated sample was divided into two portions: $9 \mathrm{~mL}$ for IMM test and $1 \mathrm{~mL}$ for the culture test. The positivity or negativity of the water samples by the IMM was visually recorded by the colorimetric endpoint reaction. The proportion of positive results by the IMM was determined for each batch of ten $100 \mathrm{~mL}$ replicates for each sample.

\section{Reference culture method}

For water testing and detection limit study, ISO11731-Part 1 was applied. Water samples were concentrated as described above. Briefly, after filtration of the volume examined, $0.1 \mathrm{~mL}$-portion of the prepared sample was spread on the surface of BCYE agar (Buffered Charcoal Yeast Extract) medium supplemented with glycine, vancomycin, polymixine and cicloheximide (GVPC medium) (bioMérieux, Spain), while a $9 \mathrm{~mL}$-portion of the prepared sample was tested by the IMM. The samples inoculated with high concentrations of L. pneumophila were first diluted with the same water matrix to ensure the count of colony forming units (CFU). The cultures were incubated for 10 days at $37 \pm 1^{\circ} \mathrm{C}$ in humid atmosphere containing $5 \%$ of $\mathrm{CO}_{2}$.

\section{Immunomagnetic technique}

The IMM test (Legipid $^{\circledR}$ Legionella Fast Detection kit, Biótica, Spain), contained different reagents (L0, L1, L2, L3, L4, L5, and L6) and an easy to handle magnetic particle concentrator comprised by a magnet and two glass cuvettes. Unless otherwise stated, aall steps were conducted at room temperature in the magnetic particle 
concentrator. Nine milliliters portions of each prepared sample for water testing and detection limit studies were transferred to the kit glass cuvette, and $1 \mathrm{~mL}$ of L1 reagent containing Legionella pneumophila-binding magnetic beads (LPBM) suspension was added. The mixture was mildly rocked for 15 minutes. LPBM separation was performed by applying a magnet to the cuvette for $5 \mathrm{mi}$ nutes, and the supernatant was discarded overturning the cuvettes. The LPBM was resuspended/washed with 5 $\mathrm{ml}$ of reagent L2 followed by magnetic separation as above. The LPBM were then incubated in $1 \mathrm{ml}$ of reagent L3 for 10 minutes, were captured with the magnet ( $3 \mathrm{~min}$ ), was resuspended/washed three times with $5 \mathrm{ml}$ of reagent L2, and were magnetically captured again (3 min). Reagent L4 includes two powder co-substrates (1.3 $\mathrm{mg}$ each one) for the colorimetric reaction and it was dissolved in $1.3 \mathrm{~mL}$ of reagent L5. The LPBM were resuspended in this solution under gentle agitation for 2 minutes to generate the signal. Then $100 \mu \mathrm{L}$ of $\mathrm{L} 6$ reagent was added to stop the reaction. The mixture was rocked for 1 minute. The LPBM were captured again as described above, and after 5 minutes, the color was compared with a negative control (without L. pneumophila). The kit is intended to provide a semi-quantitative measure of L. pneumopila concentration, by interpolation of the color developed by the tested sample in the supplied color chart. If the colorimetric reaction showed no difference between sample and negative control after two minutes, then the reaction was allowed to proceed for 10 minutes before stopping to trap low positives which correspond to an estimate level around the LOD50 of the IMM test.

A test is considered positive if at 2 minutes or before 10 minutes color difference appears with the control. A positive L. pneumophila test must have a color higher than the color control at 2 minutes from starting colorimetric reaction. Then reaction was stopped following the protocol instructions. General estimation of the level of L. pneumophila in the sample was obtained comparing the test color with the color chart. If there was no color difference at 2 minutes, the reaction was allowed continue up to 10 minutes and then it was stopped. A positive L. pneumophila test must have a color higher than the color control at 10 minutes from starting colorimetric reaction. In this case, the estimated level of L. pneumophila was low, up to two orders of magnitude $\left(10^{2} \mathrm{CFU} /\right.$ volume examined). A negative L. pneumophila test was considered if there was no color difference with the control after 10 minutes.

\section{Calculation of performance characteristics}

The test performance characteristics (specificity, sensitivity, false positives, false negatives, and efficiency) of the IMM were determined. Available ISO guides are designed to validate methods based on the microbial growth and the key issue is the "growth unit" capable to growth in a nutrient media. Although the qualitative IMM kit is not based on the growth unit, a first categorization of the presumptive results was obtained by using a two-by-two contingency table, following the scheme provided by the norm ISO/TR13843 [39]. IMM presumptive results were compared with the ones obtained with the reference method (ISO11731). These results were divided into four categories: (a) number of presumptive positives by the IMM found positive by the reference culture method (true positives), (b) number of presumptive negatives by the IMM found positive by the reference culture method (false negatives), (c) number of presumptive positives by the IMM found negative by the reference culture method (false positives), and (d) number of presumptive negatives by the IMM found negative by the reference culture method (true negatives). The sensitivity was defined as the ability of IMM to detect the target microorganism compared to the reference culture method, as follows: $(\mathrm{a} \times 100) /(\mathrm{a}+\mathrm{b})$. The specificity is defined as the inability of the IMM to detect the target microorganism when it is not detected by the reference culture method, as follows: $(\mathrm{d} \times 100) /$ $(c+d)$. Finally, the efficiency is a general single parameter, which gives the agreement between the response obtained by the IMM and the reference culture method, as follows: $(\mathrm{a}+\mathrm{d}) \times 100 / \mathrm{n}$, where $\mathrm{n}$ is the total number of tests. The percentage of false positives is calculated as $(c \times 100) /(a+c)$, and the percentage of false negatives is calculated as $(b \times 100) /(b+d)$.

A qualitative test can be used as screening assay with confirmation. Only in this case, positive presumptive result confirmed as negative by the confirming culture method can be re-categorized as true negative. Performance characteristics were also calculated with this consideration, according to the guidelines of certification bodies [40].

\section{Calculation of detection limit}

Detection limit was established as the lowest number of cultivable L. pneumophila organisms that can be detected with a probability of $50 \%$. This parameter socalled $\mathrm{LOD}_{50}$ is estimated using a statistical model (Spearman-Kärber test) but not directly measured $[37,38,40]$.

\section{Collaborative trial}

A collaborative trial involving twelve independent laboratories was performed to evaluate the validity of the IMM by testing identical samples. The collaborative trial was designed and conducted according to internationally accepted guidelines [37,41-49]. It has been shown that 
concentration methods can have highly variable recovery rates, making difficult to obtain identical samples especially for low concentrations of L. pneumophila [50]. Since the objective was the evaluation of the detection part of the IMM, the tested sample simulated the concentrated sample that is habitually obtained in the laboratory from an original sample, thus avoiding the concentration phase.

In this collaborative trial, a microbiological reference material in pill format was used (BaCuanti, Labaqua, Spain). According to the manufacturer's instructions, water samples were obtained by diluting these pills. The twelve participating laboratories received pills of $L$. pneumophila at four levels: (i) pills $\mathrm{P}_{6}$ and $\mathrm{P}_{8}$ as negative control, (ii) pills $\mathrm{P}_{1}$ and $\mathrm{P}_{3}$, containing a medium level of $L$. pneumophila, (iii) pills $\mathrm{P}_{2}, \mathrm{P}_{5}$ and $\mathrm{P}_{9}$, containing a high level of $L$. pneumophila, and (iv) pills $\mathrm{P}_{4}$ and $\mathrm{P}_{7}$, containing a low level of L. pneumophila.

To minimize any interlaboratory variability, all the required reagents were purchased from Biótica, Bioquímica Analítica S. L. Each participant received a detailed protocol describing the culture technique, the immunomagnetic run, and a reporting form to record the obtained results.

\section{Samples preparation}

The pills were supplied to the participating laboratories into individual sealed vials. For sample reconstitution, the safety seal was removed under aseptic conditions, the vial was opened, and $20 \mathrm{~mL}$ sterile distilled water were added, allowing to mix for $10 \mathrm{~min}$ at room temperature, gently shaking every $2 \mathrm{~min}$. Sterile water was added up to a final volume of $100 \mathrm{~mL}$. Then, three serial decimal dilutions $\left(10^{-1}, 10^{-2}\right.$, and $\left.10^{-3}\right)$ of each sample were prepared.

\section{Reference culture method}

Determination of $L$. pneumophila by culture isolation was conducted in accordance with the ISO 11731-Part 2. Five milliliters of each sample, as well as its corresponding 10fold serial dilutions were filtered through cellulose ester membranes (11406-47-ACN; Sartorius, Germany). The membranes were placed on the surface of the BCYE- $\alpha+$ GVPC medium (bioMérieux; Spain) and were incubated at $37^{\circ} \mathrm{C}$, preferably in a $5 \% \mathrm{CO} 2$ atmosphere for a period between 5 and 10 days.

\section{Immunomagnetic technique}

Analysis using the IMM test kit was performed in accordance to the protocol described previously. Results were reported as presence/absence in $9 \mathrm{~mL}$, and the aproximate concentrations of L. pneumophila were estimated by intercalation of the end-point colour developed in the analysed sample in the colour chart provided by the manufacturer. Accordingly, samples similar to the negative control one were labelled as $<$ LOD (limit of detection), colour between the negative control and the first colour mark corresponded to $10^{2}-10^{3} \mathrm{CFU} / 9 \mathrm{~mL}$, colour similar to the first colour mark corresponded to $10^{3}$ CFU/9 mL, colour between first and the second colour mark corresponded to $10^{3}-10^{4} \mathrm{CFU} / 9 \mathrm{~mL}$, colour similar to the second colour mark corresponded to $10^{4} \mathrm{CFU} / 9 \mathrm{~mL}$, and colour darker than the second colour mark was indicative of $>10^{4} \mathrm{CFU} / 9 \mathrm{~mL}$.

\section{Statistical data analysis}

The results reported by eleven of the twelve participating laboratories were evaluated following statistical methods described in the ISO/DIS 13528. One laboratory was rejected due to incorrect application of the trial protocol.

\section{Competing interests}

Financial competing interests: GR and BB are employed at Biótica from which test for Legionella detection was supplied. The author(s) declare that there are no competing interests. Non-financial competing interests: The authors declare that there are no non-financial competing interests.

\section{Authors' contributions}

GR and RF conceived the study. IS, BB, GR designed the experiments. RF and GR wrote the paper. IS, BB, SM performed experiments and analyzed data. RF and $E B$ helped with research design. IS, SM, RF, GR helped with manuscript discussion. IS provided samples. RF, EB helped to draft the manuscript. All authors have read and approved the final manuscript.

\section{Acknowledgements}

Authors are indebted to Dr. Ángel Berenguer (Instituto de Materiales, Universidad de Alicante) for critical reading of the manuscript. Inma Solís is indebted to Dr. Juan José Borrego (Spanish Society for Microbiology) for fruitful discussions. Guillermo Rodríguez is indebted to Dr. V. Catalán for fruitful technical cooperation in collaborative trial. This study was funded by the Centre for the Development of Industrial Technology (Programme NEOTEC) and Genoma España Foundation, from the Spanish Ministry of Science and Innovation, and also by the Institute for Small and Medium Industry of the Generalitat Valenciana (IMPIVA) attached to Spanish Ministry of Industry.

\section{Author details}

'Biótica, Bioquímica Analítica, S.L, Science and Technology Park of Jaume University, Campus Riu Sec - Espaitec 2, planta baja, E12071, Castellón de la Plana, Spain. ${ }^{2}$ Iproma, S.L, Cno.de la Raya 46, 12005, Castellón, Spain. ${ }^{3}$ Departamento de Biocatálisis, Instituto de Catálisis y Petroleoquímica, Consejo Superior de Investigaciones Científicas, Campus UAM-CSIC, 28049, Cantoblanco, Madrid, Spain. ${ }^{4}$ Institut de Microelectrònica (IMB-CNM, CSIC), Campus Univ. Autònoma de Barcelona, 08193, Bellaterra, Barcelona, Spain.

Received: 15 November 2012 Accepted: 16 April 2013 Published: 22 April 2013

\section{References}

1. Helbig JH, Kurtz JB, Pastoris MC, Pelaz C, Luck PC: Antigenic lipopolysaccharide components of Legionella pneumophila recognized by monoclonal antibodies: possibilities and limitations for division of the species into serogroups. J Clin Microbiol 1997, 35:2841-2845.

2. Yu VL, Plouffe JF, Pastoris MC, Stout JE, Schousboe M, Widmer A, Summersgill J, File T, Heath CM, Paterson DL, Chereshsky A: Distribution of Legionella species and serogroups isolated by culture in patients with sporadic community- acquired pneumonia: an international collaborative survey. $J$ Infect Dis 2002, 186:127-128. 
3. Tijet $\mathrm{N}$, Tang $\mathrm{P}$, Romilowych M, Duncan C, Ng V, Fisman DN, Jamieson F, Low DE, Guyard C: New endemic Legionella pneumophila serogroup 1 clones, Ontario, Canada. Emerg Infect Dis 2010, 16:447-454

4. Sabrià M, Campins M: Legionnaires' Disease: Update on Epidemiology and Management Options. Am J Respir Crit Care Med 2003, 2:235-243.

5. Fields BS, Benson RF, Besser RE: Legionella and Legionnaires' disease: 25 Years of Investigation. Clin Microbiol Rev 2002, 15:506-526.

6. Fliermans CB, Cherry WB, Orrison LH, Smith SJ, Tison DL, Pope DH: Ecological distribution of Legionella pneumophila. Appl Environ Microbiol 1981, 41:9-16.

7. Colbourne JS, Dennis PJ, Trew RM, Berry G, Vesey G: Legionella and public water supplies. Water Sci Technol 1988, 20:11-20.

8. Steinert M, Hentschel U, Hacker J: Legionella pneumophila: an aquatic microbe goes astray. FEMS Microbiol Rev 2002, 26:149-162.

9. Mampel J, Spirig T, Weber SS, Haagensen JAJ, Molin S, Hilbi H: Planktonic Replication Is Essential for Biofilm Formation by Legionella pneumophila in a Complex Medium under Static and Dynamic Flow Conditions. Appl Environ Microbiol 2006, 72:2885-2895.

10. Ragull S, Garcia-Nuñez M, Pedro-Botet ML, Sopena N, Esteve M, Montenegro R, Sabrià M: Legionella pneumophila in Cooling Towers: Fluctuations in Counts, Determination of Genetic Variability by Pulsed-Field Gel Electrophoresis (PFGE), and Persistence of PFGE Patterns. Appl Environ Microbiol 2007, 73:5382-5384

11. Wéry N, Bru-Adan V, Minervini C, Delgénes JP, Garrelly L, Godon JJ: Dynamics of Legionella spp. and Bacterial Populations during the Proliferation of L. pneumophila in a Cooling Tower Facility. App/ Environ Microbiol 2008, 74:3030-3037

12. Moritza MM, Flemminga HC, Wingender J: Integration of Pseudomonas aeruginosa and Legionella pneumophila in drinking water biofilms grown on domestic plumbing materials. Int J Hyg Environ Health 2010, 213:190-197.

13. Bej AK, Mahbubani MH, Atlas RM: Detection of viable Legionella pneumophila in water by polymerase chain reaction and gene probe methods. App/ Environ Microbiol 1991, 57:597-600.

14. García MT, Jones S, Pelaz C, Millar RD, Abu KY: Acanthamoeba polyphaga resuscitates viable non-culturable Legionella pneumophila after disinfection. Environ Microbiol 2007, 9:1267-1277.

15. Alleron L, Merlet N, Lacombe C, Frère J: Long-term survival of Legionella pneumophila in the viable but nonculturable state after monochloramine treatment. Curr Microbiol 2008, 57:497-502.

16. Cunliffe DA: Inactivation of Legionella pneumophila by monochloramine. J Appl Bacteriol 1990, 68:453-459.

17. Kool JL, Carpenter JC, Fields BS: Effect of monochloramine disinfection of municipal drinking water on risk of nosocomial Legionnaires' disease. Lancet 1999, 353:272-277.

18. Kuchta JM, States SJ, McNamara AM, Wadowsky RM, Yee RB: Susceptibility of Legionella pneumophila to chlorine in tap water. Appl Environ Microbiol 1983, 46:1134-1139.

19. International Organization for Standardization: ISO 11731:1998 Water qualitydetection and enumeration of Legionella. Geneva-Switzerland; 1998.

20. International Organization for Standardization: ISO 11731-2:2004 Water quality - Detection and enumeration of Legionella - Part 2: Direct membrane filtration method for waters with low bacterial counts. Geneva-Switzerland; 2004.

21. Hussong D, Colwell RR, O'Brien M, Weiss E, Pearson AD, Weiner RM, Burge WD: Viable Legionella pneumophila not detectable by culture on agar media. Viable Legionella pneumophila not detected by culture on agar media. Biotechnol 1987, 5:947-950.

22. Yanez MA, Carrasco-Serrano C, Barbera VM, Catalán V: Quantitative Detection of Legionella pneumophila in Water Samples by Immunomagnetic Purification and Real-Time PCR Amplification of the dotA Gene. Appl Environ Microbiol 2005, 71:3433-3441.

23. Yanez MA, Carrasco-Serrano C, Barbera VM, Catalán V: Validation of a new seminested PCR-based detection method for Legionella pneumophila. J Microbiol Meth 2007, 70:214-217.

24. Dusserre E, Ginevra C, Hallier-soulier S, Festoc G, Etienne J, Jarraud S: A PCRBased Method for Monitoring Legionella pneumophila in Water Samples Detects Viable but Noncultivable Legionellae That Can Recover Their Cultivability. Appl Environ Microbiol 2008, 74:4817-4824.

25. Buchbinder S, Trebesius K, Heesemann J: Evaluation of detection of Legionella spp. in water samples by fluorescence in situ hybridization,
PCR amplification and bacterial culture. International J Med Microbiol 2002, 292:241-245.

26. Amann R, Ludwig W: Ribosomal RNA-targeted nucleic acid probes for studies in microbial ecology. FEMS Microbiol Rev 2000, 24:555-565.

27. Lazcka O, Del Campo FJ, Muñoz X: Pathogen detection: A perspective of traditional methods and biosensors. Biosens Bioelectron 2007, 22:1205-1217.

28. Brooks BW, Devenish J, Lutze-Wallace CL, Milnes D, Robertson RH, BerlieSurujballi G: Evaluation of a monoclonal antibody-based enzyme-linked immunosorbent assay for detection of Campylobacter fetus in bovine preputial washing and vaginal mucus samples. Vet Microbiol 2004, 103:77-84.

29. Satoh W, Nakata M, Yamamoto H, Ezaki T, Hiramatsu K: Enumeration of Legionella CFU by colony hybridization using specific DNA probes. Appl Environ Microbiol 2002, 68:6466-6470.

30. Aurell H, Catala P, Farge P, Wallet F, Le Brun M, Helbig JH, Jarraud S, Lebaron P: Rapid detection and enumeration of Legionella pneumophila in hot water systems by solid-phase cytometry. Appl Environ Microbiol 2004, 70:1651-1657.

31. Olsvik Ø, Popovic T, Skjerve E, Cudjoe KS, Hornes E, Ugelstad J, Uhlen M: Magnetic separation techniques in diagnostic microbiology. Clin Microbiol Rev 1994, 7:43-54.

32. Gehring AG, Irwin PL, Reed SA, Tu SI, Andreotti PE, Akhavan-Tafti H, Handley RS: Enzyme-linked immunomagnetic chemiluminescent detection of Escherichia coli 0157:H7. J Immunol Meth 2004, 293:97-106.

33. Füchslin HP, Kötzsch S, Egli T: Rapid and quantitative detection of Legionella pneumophila applying immunomagnetic separation and flow cytometry. Cytometry A 2010, 77(3):264-274.

34. Keserue HA, Baumgartner A, Felleisen R, Egli T: Rapid detection of total and viable Legionella pneumophila in tap water by immunomagnetic separation, double fluorescent staining and flow cytometry. Microb Biotechnol 2012, 5:753-763.

35. Rodríguez $\mathrm{G}$, Bedrina $\mathrm{B}$, Jiménez M: Validation of the Legipid ${ }^{\circledR}$ Bioalarm Legionella Assay. J AOAC Int 2012, 95:1440-1451.

36. Borella P, Montagna MT, Stampi S, Stancanelli G, Romano-Spica V, Triassi M Marchesi I, Bargellini A, Tatò D, Napoli C, Zanetti F, Leoni E, Moro M, Scaltriti S: Ribera D'Alcalà G, Santarpia R, Boccia S: Legionella Contamination in Hot Water of Italian Hotels. App/ Environ Microbiol 2005, 71:5805-5813.

37. Association française de normalisation (AFNOR): Application à l'analyse microbiologique de l'eau, Protocole de Validation d'une méthode alternative commerciale par rapport à une méthode de référence. France; 2010.

38. NordVal: Protocol for the validation of alternative microbiological methods. Oslo-Norway; 2009

39. International Organization for Standardization: ISO/TR 13843:2000(E) Water quality - Guidance on validation of microbiological methods. GenevaSwitzerland; 2000.

40. Feldsine P, Abeyta C, Andrews WH: AOAC International Methods Committee Guidelines for Validation of Qualitative and Quantitative Food Microbiological Official Methods of Analysis. J AOAC Int 2002, 85:1187-1200.

41. International Laboratory Accreditation Cooperation: ILAC- G13:08/2007 ILAC Guidelines for Requirements for the Competence of Provides of Proficiency Testing Schemes. Silverwater-Australia; 2007.

42. International Organization for Standardization: ISO5725-6:1994 Accuracy (trueness and precision) of measurement methods and results-Part 6: Use in practice of accuracy values. Geneva-Switzerland; 1994.

43. International Organization for Standardization: ISO 8199:2005 Water qualityGeneral guidance on the enumeration of micro-organisms by culture. GenevaSwitzerland; 2005.

44. International Organization for Standardization: ISO 13528:2005 Statistical methods for use in proficiency testing by interlaboratory comparisons. Geneva-Switzerland; 2005.

45. International Organization for Standardization: ISO 7218:2007 Microbiology of food and animal feeding stuffs-General requirements and guidance for microbiological examinations. Geneva-Switzerland; 2007.

46. International Organization for Standardization: ISO/IEC 17043:2010 Conformity assessment-General requirements for proficiency testing. Geneva-Switzerland; 2010.

47. National Accreditation Entity (ENAC): CGA-ENAC-PPI:2003 General criteria for accreditation of testing proficiency schemes suppliers according UNE 66543-1 and ILAC-G13 guide. Madrid-Spain; 2003.

48. National Accreditation Entity (ENAC): G-ENAC-14: 2008 Guide for participation in intercomparison exercises. Madrid-Spain; 2008. 
49. Spanish Association for Standarization and Certification (AENOR): UNE 66543-1:1999 IN. 1999 Proficiency Testing By Interlaboratory Comparisons. Part 1: Development and Operation of Proficiency Testing Schemes. Madrid-Spain; 1999.

50. Boulanger CA, Edelstein PH: Precision and Accuracy of Recovery of Legionella pneumophila from Seeded Tap Water by Filtration and Centrifugation. Appl Environ Microbiol 1995, 61:1805-1809.

doi:10.1186/1471-2180-13-88

Cite this article as: Bedrina et al:: Fast immunosensing technique to detect Legionella pneumophila in different natural and anthropogenic environments: comparative and collaborative trials. BMC Microbiology 2013 13:88.

\section{Submit your next manuscript to BioMed Central and take full advantage of:}

- Convenient online submission

- Thorough peer review

- No space constraints or color figure charges

- Immediate publication on acceptance

- Inclusion in PubMed, CAS, Scopus and Google Scholar

- Research which is freely available for redistribution 